\title{
Diversidade da macrofauna Edáfica em um fragmento de Floresta Estacional Semidecidual, Paraná
}

A fauna do solo ou fauna edáfica, compreende os invertebrados que vivem no solo durante toda a vida ou em algum estágio do ciclo biológico. A macrofauna é constituída pelos invertebrados com mais de $10 \mathrm{~mm}$ de comprimento ou com mais de $2 \mathrm{~mm}$ de diâmetro corporal e tem participação direta na dinâmica ecossistêmica, em processos de ciclagem de nutrientes e na estrutura e qualidade física do solo. Este trabalho objetivou verificar a diversidade da macrofauna do solo em um fragmento de Floresta Estacional Semidecidual Montana no município de Corumbataí do Sul, Paraná, abrangendo os meses de agosto a dezembro de 2014. Foram realizadas coletas mensais de solo (três estratificações de profundidade) e de serapilheira pelo método TSBF, esta última acrescida de capturas por meio de armadilhas do tipo 'pitfall-traps', com posterior quantificação da fauna e identificação dos grupos taxonômicos. Foram encontrados 3572 indivíduos de macroinvertebrados no solo do fragmento, distribuídos em 16 unidades taxonômicas. O grupo mais abundante tanto em serapilheira quanto no solo foi Hymenoptera: Formicidae, como também ao longo dos meses. A diversidade da macrofauna variou ao longo dos meses e ao longo dos estratos do sistema soloserapilheira revelando papel sazonal e espacial na diversidade da macrofauna.

Palavras-chave: Sistema Solo-Serapilheira; Invertebrados de Solo; Sistema Agroflorestal.

\section{Edaphic macrofauna diversity in a fragment of Semideciduous Seasonal Forest, Paraná}

\begin{abstract}
The fauna of the soil or edaphic fauna comprises the invertebrates that live in the soil throughout their life or at some stage of the biological cycle. The macrofauna consists of invertebrates with more than $10 \mathrm{~mm}$ in length or more than $2 \mathrm{~mm}$ of body diameter and has a direct participation in the ecosystem dynamics, in nutrient cycling processes and in the structure and physical quality of the soil. This work aimed to verify the soil macrofauna diversity in a fragment of Seasonal Semideciduous Montana Forest in the county of Corumbataí do Sul, Paraná, from August to December 2014. Soil samples were collected monthly (three depth stratifications) and of litter by the TSBF method, the latter being increased by means of pitfall traps, with subsequent quantification of the fauna and identification of the taxonomic groups. 3572 individuals of macroinvertebrates were found in the soil of the fragment, distributed in 16 taxonomic units. The most abundant group in both litter and soil was Hymenoptera: Formicidae, as well as throughout the months. Macrofauna diversity varied over the months and throughout the strata of the soil-litter system revealing seasonal and spatial role in the macrofauna diversity.
\end{abstract}

Keywords: Soil-Litter System; Soil Invertebrates; Agroforestry System.

Topic: Ciências do Solo

Reviewed anonymously in the process of blind peer
Received: $10 / 08 / 2018$

Approved: 24/09/2018
Tamyris Tavares da Silva (it

Universidade Tecnológica Federal do Paraná, Brasil http://lattes.cnpq.br/1243986358826489

http://orcid.org/0000-0002-5674-0687

tamyris_tavares@hotmail.com

Raquel de Oliveira Bueno (ii)

Universidade Tecnológica Federal do Paraná, Brasil http://lattes.cnpq.br/5967815195662473

http://orcid.org/0000-0002-9917-5147

quelloliveira@gmail.com

Luciane Maria Vieira do Couto

Universidade Tecnológica Federal do Paraná, Brasil

http://lattes.cnpq.br/1597388093205327

http://orcid.org/0000-0002-7801-3130

lucianeutfpr@gmail.com

\author{
Débora Cristina de Souza (it) \\ Universidade Tecnológica Federal do Paraná, Brasil \\ http://lattes.cnpq.br/9682347849778341 \\ http://orcid.org/0000-0001-6916-6330 \\ dcsouza@gmail.com \\ Paulo Agenor Alves Bueno (iD \\ Universidade Tecnológica Federal do Paraná, Brasil \\ http://lattes.cnpq.br/1838875919713622 \\ http://orcid.org/0000-0002-8929-1400 \\ paabueno@gmail.com
}

Referencing this:

SILVA, T. T.; BUENO, R. O.; COUTO, L. M. V.; SOUZA, D. C.; BUENO, P. A. A.. Diversidade da macrofauna Edáfica em um fragmento de Floresta Estacional Semidecidual, Paraná. Revista Ibero Americana de Ciências Ambientais, v.9, n.7, p.34-41, 2018. DOI: http://doi.org/10.6008/CBPC2179-6858.2018.007.0004 


\section{INTRODUÇÃO}

Solos são constituídos de água, gases, material mineral e diversos organismos juntamente com muitos tipos de matérias de origem biológica (LIMA et al., 2007). O Brasil possui uma das maiores biodiversidades do planeta, sendo a fauna de solo importante componente dessa diversidade. Ocorre dentro do solo ou na serapilheira e gera importantes serviços ambientais, que são pouco reconhecidos e valorizados (SILVA et al., 2012).

A diversidade da fauna de solo tem relação com uma extensa variedade de recursos e microhabitats que o solo juntamente com a serapilheira pode ofertar, oferecem um aglomerado de condições microclimáticas, auxiliando vários grupos funcionais que são associados. Assim, o sistema solo-serapilheira serve como moradia natural para uma vasta diversidade de organismos e microrganismos, que possuem tamanhos e metabolismo diferentes, responsáveis por muitas atividades (MOÇO et al., 2005).

Os organismos que compõem o solo são classificados de acordo com o tamanho corporal. Organismos com mais de $10 \mathrm{~mm}$ de comprimento ou com mais de $2 \mathrm{~mm}$ de diâmetro corporal constituem a macrofauna edáfica, sendo os maiores invertebrados da fauna de solo (SILVA et al., 2006). A maior parte dos animais de solo ocorre em até $30 \mathrm{~cm}$ de profundidade (RUIZ et al., 2008). Os invertebrados da macrofauna de solo têm o papel de fragmentação do material orgânico e regula a população microbiana responsável pela mineralização e produção de húmus, influenciando na reciclagem de matéria orgânica e disponibilizando nutrientes que são absorvidos pelas plantas (SILVA et al., 2006).

Informações sobre a macrofauna de solo em florestas são importantes devido às funções que esta pode exercer neste ecossistema. Esses organismos contribuem para a melhoria da estrutura e qualidade física do solo, através da movimentação de partículas dentro e entre os horizontes, da formação de agregados e consequente aumento da resistência do solo à erosão (LAVELLE et al., 2006). Participam da decomposição de matéria orgânica, da mineralização e disponibilização de nutrientes para as plantas (LAVELLE et al., 2006), sendo por isso, sua presença considerada indicativo de solo saudável (SCHIEDECK et al., 2009; LIMA et al., 2010).

Áreas agrícolas com florestas próximas tendem a melhorar os parâmetros físicos e químicos do solo, preservando principalmente suas características biológicas. Estudos sobre a Biologia do Solo tem tido um interesse especial dos pesquisadores das ciências agrárias nos últimos anos (SILVA et al., 2006; ANDERSON, 2009; LIMA et al., 2010; ARAÚJO et al., 2015), no entanto alguns campos ainda carecem ser mais estudados sobretudo nos domínios da Floresta Estacional Semidecidual (MENEZES et al., 2009; MARQUES, 2014; PINHEIRO et al., 2014). O objetivo desse trabalho foi verificar a diversidade da macrofauna edáfica presente em um fragmento de Floresta Estacional Semidecidual Montana situado em uma propriedade rural de Corumbataí do Sul, em estratos de solo e na serapilheira.

\section{MATERIAIS E MÉTODOS}

O presente estudo foi realizado em fragmento florestal situado no município de Corumbataí do Sul,

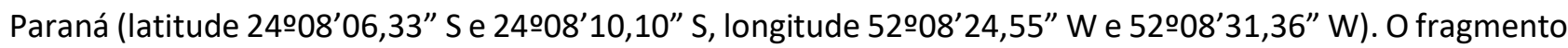


possui em torno de 16 hectares de área de Floresta Estacional Semidecidual Montana, com alta declividade e indícios de atividade humana de desmatamento seletivo. Está circundado por pastagens e plantações que caracterizam atividades de agricultura familiar, preponderantes dessa região.

A região de Corumbataí do Sul pertence ao clima subtropical úmido mesotérmico com verões quentes e geadas pouco frequentes, com tendência de concentração das chuvas nos meses de verão, sem estação seca definida. A média das temperaturas dos meses mais quentes é superior a $22{ }^{\circ} \mathrm{C}$ e a dos meses mais frios, inferior a $18^{\circ} \mathrm{C}$. Os índices pluviométricos apresentam-se em média entre $1.600 \mathrm{~mm}$ e $1.800 \mathrm{~mm}$ por ano.

O município de Corumbataí do Sul está inserido no Terceiro Planalto Paranaense e a classe de declividade predominante está entre 12 e 30\%. Em relação ao relevo, apresenta gradiente de 900 metros, com altitudes variando entre 320 (mínima) e 1220 (máxima) metros sobre o nível do mar (MINEROPAR, 2006). Apresenta predominantemente o Neossolo Litólico Eutrófico que compreende solos rasos e normalmente associados a relevos mais íngremes (EMBRAPA, 2007). A coleta do solo para a retirada dos macroinvertebrados do solo foi realizada mensalmente de agosto a dezembro de 2014 , totalizando cinco meses de coletas. Foram estabelecidos três pontos dentro do fragmento (Figura 1), entre as altitudes de 650 a 710 metros, na região média vertente do relevo.

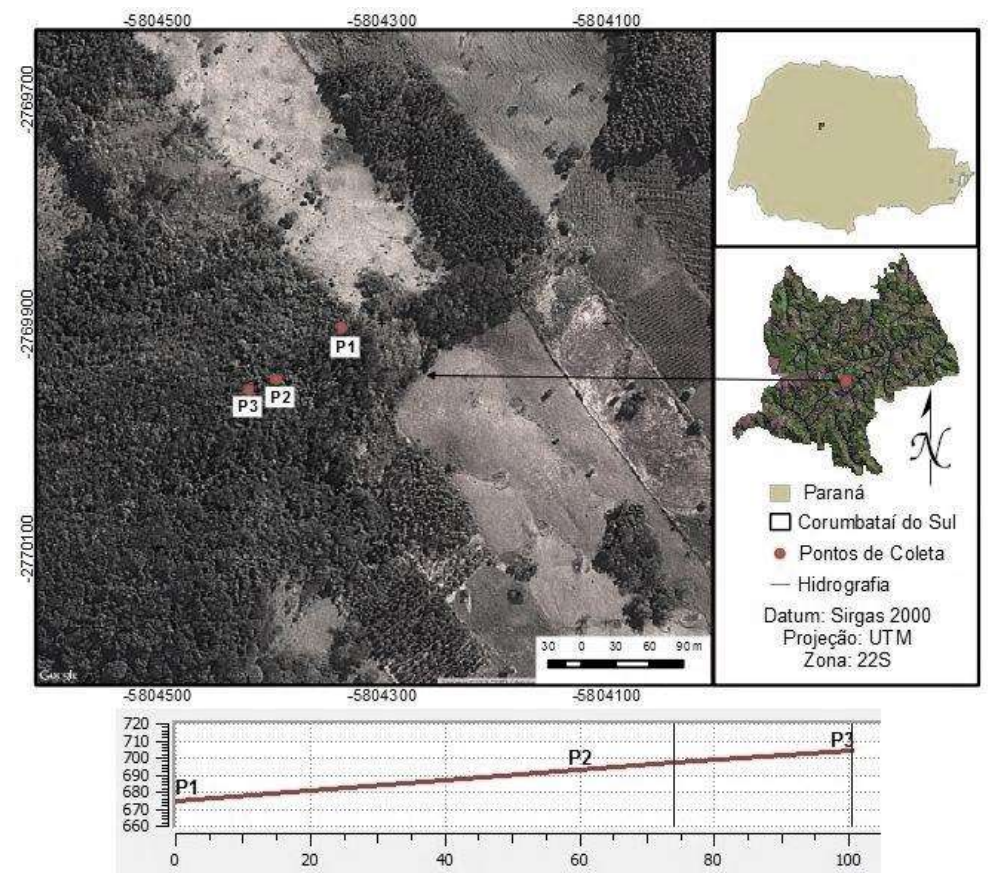

Figura 1: Localização dos pontos de coleta demarcados na imagem Land Sat, cena 222/77, de 07 de maio de 2015 e altitudes dos pontos de coleta, no município de Corumbataí do Sul, Paraná.

A coleta dos macroinvertebrados foi baseada no método do TSBF (Tropical Soil Biology and Fertility) (ANDERSON et al., 1993), método padronizado segundo ISO 23611-5: 2011, sendo que uma área de 25 × 25 $\mathrm{cm}$ foi demarcada com estacas da qual foi retirada a serapilheira e o solo em três estratos de profundidade, de $0-10 \mathrm{~cm}$, de $10-20 \mathrm{~cm}$, e de $20-30 \mathrm{~cm}$. Assim foram obtidos quatro estratos mensais de cada ponto de coleta. A extração dos animais foi realizada em laboratório onde todos os invertebrados visíveis a olho nu 
foram retirados e acondicionados em potes contendo álcool 70\%. Os macroinvertebrados foram contados e agrupados em unidades taxonômicas abrangentes.

Foram instaladas armadilhas do tipo 'pitfall-trap' em cada um dos pontos, para captura de macroinvertebrados, com a finalidade de avaliar a composição da fauna epígea, ou seja, dos invertebrados que atuam principalmente na superfície do solo. Recipientes com cerca de $10 \mathrm{~cm}$ de altura e $10 \mathrm{~cm}$ de diâmetro foram dispostos no solo de tal forma que os animais caíssem acidentalmente. Cada recipiente recebeu cerca de $100 \mathrm{ml}$ de formol $4 \%$ para que os animais não fugissem e pudessem ser conservados, pois permaneceram no campo durante 30 dias, aproximadamente. Após esse tempo, os recipientes foram recolhidos e levados ao laboratório para contagem e identificação dos animais. Larvas e adultos foram separados, pois apresentam papel funcional diferenciado no ambiente (RUIZ et al., 2008).

Para o cálculo da diversidade das comunidades de macroinvertebrados nos diferentes habitats foi utilizado o índice de Shannon-Wiener, obtido a partir do programa Bioestat versão 5.0 (AYRES et al., 2007). Foram feitas comparações entre os valores dos índices de Shannon-Wiener utilizando-se teste $T$ Hutcheson (Hutcheson, 1970), para os meses e para o sistema solo-serapilheira, com nível de significância de 5\%.

\section{RESULTADOS E DISCUSSÃO}

Foram encontrados 3572 indivíduos de macroinvertebrados no solo do fragmento, distribuídos em 16 unidades taxonômicas, considerando-se os animais capturados nas armadilhas 'pitfall' e os coletados da serapilheira e do solo. A composição relativa da comunidade de macrofauna apresentou a unidade taxonômica Hymenoptera: Formicidae com maior número de indivíduos $(64,92 \%)$, seguida de Coleoptera (8,68 \%) e Aracnida (6,55 \%) (Tabela 1). Foram encontrados 63 indivíduos larvais de Coleoptera (25,5\% dos indivíduos deste grupo), 41 de Diptera $(29,1 \%)$ e 12 de Lepidoptera (100\%).

A macrofauna pode ser classificada em três grupos funcionais, os engenheiros do ecossistema, os decompositores e os micropredadores, sendo que os fatores que delimitam essa classificação dependem do seu tamanho, natureza da estrutura que criam no solo, além do principal tipo de relação que desenvolvem com os microrganismos (AQUINO et al., 2008). Animais Hymenoptera: Formicidae são importantes nos processos de degradação de matéria orgânica nos ecossistemas, quebrando o material vegetal em frações menores e facilitando a ação decompositora dos microrganismos. Destaca-se o papel funcional desse grupo de invertebrados nos ciclos biogeoquímicos dos sistemas florestais e em diversos ecossistemas (MAYER et al., 2005; FEENER JR et al., 2008). Agem na formação e estruturação do solo, atuando como engenheiros do solo (MELO et al., 2009). Em floresta essa predominância de Hymenoptera: Formicidae pode estar relacionada à proximidade de sistemas agrícolas que estão no entorno do fragmento, onde o efeito de sistemas agrícolas pode influenciar na composição da fauna de solo, bem como aumentar a frequência de alguns grupos, como o de formigas, em virtude da disponibilidade de recursos e ação antrópica (ANDERSON, 2009). 
Tabela 1: Abundância de macroinvertebrados capturados por armadilhas 'pitfall', retirados de serapilheira e estratos de solo em fragmento de Floresta Estacional Semidecidual, Corumbataí do Sul, Paraná.

\begin{tabular}{|c|c|c|c|c|c|c|c|}
\hline & $\begin{array}{ll}\text { Abundância } & \text { de } \\
\text { Macroinvertebrados } & \end{array}$ & & & & & & \\
\hline Unidade Taxonômica & Pitfall & $\begin{array}{l}\text { Serapilheir } \\
\text { a }\end{array}$ & $\begin{array}{l}\text { Estrato } \\
1\end{array}$ & $\begin{array}{l}\text { Estrato } \\
2\end{array}$ & $\begin{array}{l}\text { Estrato } \\
3\end{array}$ & $\begin{array}{l}\text { Total1 } \\
*\end{array}$ & $\begin{array}{l}\text { Total2 } \\
* *\end{array}$ \\
\hline Aracnida & 98 & 55 & 56 & 19 & 6 & 136 & 234 \\
\hline Blattodea & 1 & 4 & 2 & 2 & 2 & 10 & 11 \\
\hline Chilopoda & 3 & 7 & 29 & 11 & 3 & 50 & 53 \\
\hline Coleoptera/Larva & $218 / 11$ & $12 / 10$ & $10 / 20$ & $5 / 14$ & $2 / 8$ & $29 / 52$ & $247 / 63$ \\
\hline Collembola & 15 & 0 & 0 & 0 & 0 & 0 & 15 \\
\hline Diplopoda & 22 & 38 & 80 & 15 & 14 & 147 & 169 \\
\hline Diptera/Larva & $138 / 6$ & $1 / 15$ & $1 / 10$ & $1 / 7$ & $0 / 3$ & $3 / 35$ & $141 / 41$ \\
\hline Gastropoda & 1 & 19 & 71 & 42 & 21 & 153 & 154 \\
\hline Hemiptera & 1 & 0 & 1 & 0 & 0 & 1 & 2 \\
\hline Homoptera & 1 & 0 & 0 & 0 & 0 & 0 & 1 \\
\hline $\begin{array}{l}\text { Hymenoptera: } \\
\text { Formicidae }\end{array}$ & 1230 & 117 & 686 & 221 & 65 & 1089 & 2319 \\
\hline Isopoda & 4 & 9 & 13 & 3 & 3 & 28 & 32 \\
\hline Isoptera & 0 & 0 & 0 & 13 & 0 & 13 & 13 \\
\hline Lepidoptera/Larva & $0 / 8$ & $0 / 2$ & $0 / 2$ & 0 & 0 & $0 / 4$ & $0 / 12$ \\
\hline Oligochaeta & 0 & 1 & 9 & 3 & 2 & 15 & 15 \\
\hline Orthoptera & 49 & 1 & 0 & 0 & 0 & 1 & 50 \\
\hline Abundância Total & 1806 & 291 & 990 & 356 & 129 & 1766 & 3572 \\
\hline Número de taxa & 14 & 12 & 12 & 11 & 10 & - & - \\
\hline Diversidade & 1.14 & 1.81 & 1.20 & 1.42 & 1.61 & - & - \\
\hline
\end{tabular}

Abundância total: * sem pitfall ; ** com pitfall.

Animais dos grupos Coleoptera e Aracnida também são atuantes diretos na dinâmica dos ecossistemas florestais. Os primeiros, além de participarem da fragmentação de matéria orgânica, participam na humificação e mineralização do solo, papel funcional mantenedor de produtividade de solos agroflorestais e indicam saúde do solo quando presentes (MOÇO et al., 2005; TEIXEIRA et al., 2009). Os aracnídeos tem papel funcional ligado mais diretamente à predação e fragmentação de matéria orgânica resultantes dessa ação. Os predadores têm papel fundamental nas cadeias tróficas terrestres, alimentando-se respectivamente, de uma grande quantidade de outros artrópodes e de plantas. Por sua vez, todos estes grupos, em maior ou menor escala, integram as cadeias alimentares de numerosos grupos de vertebrados e até de algumas plantas, designadas genericamente por carnívoras ou insetívoras (SILVA et al., 2012). Entre os grupos de menor abundância relativa nesse trabalho, os Diptera podem ser evidenciados por seu papel mais evidente na decomposição de matéria orgânica morta nas fases larvais e adultas em muitos grupos. A ordem Diptera tem maior expressividade quando se trata de decomposição de cadáveres, colonizando-os em número elevado e atuam em todas as fases da decomposição (OLIVEIRA-COSTA et al., 2013).

Organismos invertebrados de solo têm sido utilizados como bioindicadores, pois revelam o estado da qualidade do solo diante das ações antrópicas. Esses organismos, de certa forma, são fáceis de serem avaliados, pois os métodos de avaliação são baseados na identificação e contagem dos indivíduos. Porém esses fatores muitas vezes são sensíveis, à medida que as populações de fauna do solo sofrem grande influência da sazonalidade e sua sobrevivência é extremamente dependente da presença de habitats específicos (ASHFORD et al., 2013).

Os índices de diversidade do sistema solo-serapilheira, como também dos meses de coleta, estão apresentados nas Tabelas 1 e 2, respectivamente. As diversidades comparadas das comunidades de 
macrofauna do sistema solo-serapilheira apresentaram diferenças, exceto entre 'pitfall' e estrato $1(t=-1,16)$, serapilheira e estrato $3(t=-1,87)$, e, estrato 2 e estrato $3(t=1,48)$ (Tabela 2$)$. A comparação das diversidades entre os meses apresentou diferença significativa, exceto entre os meses de agosto-outubro $(t=1,35)$ e setembro-outubro ( $t=-1,91)$ (Tabela 3 ). Essa diferença entre os meses pode estar associada a variáveis ambientais como temperatura média mensal e pluviosidade, como pode também estar associada às características vegetacionais e do solo devido à influência das diferentes estações do ano (LIMA et al., 2010) que, no caso do presente estudo, trata-se de inverno e primavera. Testou-se também a variação da diversidade de macrofauna nos meses em relação à pluviosidade média mensal do local, não foi verificada correlação $(r=0,37 ; \mathrm{gl}=3 ; p=0,54)$. Em ambientes de sistema florestal, o regime de chuvas é determinante para a composição da macrofauna (BARROS et al., 2008), porém com o número de meses do presente trabalho pode-se não detectar o efeito mais direto deste fator.

Tabela 2: Valores do $t$ calculado pelo teste $T$ de Hutcheson, a partir da comparação entre os índices de diversidade da macrofauna do sistema solo-serapilheira, em Corumbataí do Sul, Paraná.

\begin{tabular}{|l|l|l|l|l|l|}
\hline & \multicolumn{1}{|c|}{ Pitfall } & Serapilheira & \multicolumn{1}{|c|}{ Estrato1 } & Estrato2 & Estrato3 \\
\hline Pitfall & - & & & & \\
\hline Serrapilheira & $*-10,23$ & - & & & \\
\hline Estrato1 & $-1,16$ & $*_{-}-4,41$ & - & & \\
\hline Estrato2 & $*-3,61$ & $*_{-4,34}$ & $*_{-2,61}$ & - & \\
\hline Estrato3 & $*-4,39$ & $-1,87$ & $* 3,65$ & 1,48 & - \\
\hline
\end{tabular}

$t$ (tab) 1,$96 ;{ }^{*}$ significativamente diferentes.

Tabela 3: Valores do $t$ calculado pelo teste $T$ de Hutcheson, a partir da comparação entre os índices de diversidade da macrofauna de solo e serapilheira nos meses amostrados, em Corumbataí do Sul, Paraná.

\begin{tabular}{|l|l|l|l|l|l|}
\hline & Agosto & Setembro & Outubro & Novembro & Dezembro \\
\hline Agosto & - & & & & \\
\hline Setembro & $* 3,08$ & - & & & \\
\hline Outubro & 1,35 & $-1,91$ & - & & \\
\hline Novembro & $* 9,47$ & $* 6,28$ & $* 8,67$ & - & \\
\hline Dezembro & $* 5,94$ & $* 2,88$ & $* 4,96$ & $* 3,25$ & - \\
\hline
\end{tabular}

$t(\operatorname{tab}) 1,96 ; *$ significativamente diferentes.

Observou-se que os meses com mais indivíduos apresentaram menor diversidade (Tabela 4). Esse resultado corrobora aos de Souza et al. (2005) que encontraram a dominância de poucas espécies na comunidade, que acaba fazendo com que o aumento do número de indivíduos decresça o índice de diversidade. Nas comparações par a par com teste $T$ a maioria apresentou diferenças demonstrando que as diversidades nos meses podem ser consideradas características de cada período. Isso pode ser corroborado com os resultados obtidos por Machado et al. (2005), que observou essa mesma situação de diversidades diferentes ao longo dos meses. Pode-se ainda inferir indiretamente sobre o efeito da sazonalidade e dos ciclos biológicos dos grandes grupos que tem períodos de nascimento, eclosão e mortes definidos temporalmente.

Para a diversidade no sistema solo-serapilheira obteve-se um resultado próximo ao esperado com referência a distribuição vertical da macrofauna. Caracterizando através do estrato de ocorrência e das diversidades que se diferenciam influenciadas pela composição da fauna que é mais específica em cada estrato (ARAÚJO et al., 2015). 
Tabela 4: Abundância de macroinvertebrados coletados no sistema solo-serapilheira de um fragmento de Floresta Estacional Semidecidual, ao longo dos meses em Corumbataí do Sul, Paraná.

\begin{tabular}{|c|c|c|c|c|c|c|}
\hline & Abundância de macroinvertebrados & & & & & \\
\hline Unidade taxonômica & Agosto & Setembro & Outubro & Novembro & Dezembro & Total \\
\hline Aracnida & 20 & 32 & 42 & 18 & 25 & 137 \\
\hline Blattodea & 2 & 6 & 2 & 0 & 0 & 10 \\
\hline Chilopoda & 3 & 20 & 13 & 5 & 9 & 50 \\
\hline Coleoptera & 18 & 18 & 25 & 10 & 15 & 86 \\
\hline Diplopoda & 37 & 23 & 50 & 19 & 22 & 151 \\
\hline Diptera & 5 & 8 & 11 & 6 & 5 & 35 \\
\hline Gastropoda & 28 & 21 & 25 & 49 & 31 & 154 \\
\hline Hemiptera & 1 & 0 & 0 & 0 & 0 & 1 \\
\hline Hymenoptera Formicidae & 63 & 174 & 156 & 315 & 181 & 889 \\
\hline Isopoda & 4 & 3 & 12 & 4 & 5 & 28 \\
\hline Isoptera & 0 & 13 & 0 & 0 & 0 & 13 \\
\hline Lepidoptera & 0 & 0 & 2 & 2 & 0 & 4 \\
\hline Oligochaeta & 8 & 0 & 5 & 2 & 0 & 15 \\
\hline Orthoptera & 1 & 0 & 2 & 0 & 0 & 3 \\
\hline Abundância total & 190 & 318 & 345 & 430 & 293 & 1576 \\
\hline Número de taxa & 12 & 10 & 12 & 10 & 8 & - \\
\hline Diversidade & 1.91 & 1.61 & 1.78 & 1.04 & 1.34 & - \\
\hline
\end{tabular}

\section{CONCLUSÕES}

Os grupos taxonômicos mais abundantes são Hymenoptera Formicidae, Coleoptera e Aracnida com diversidade relativamente alta como em outros solos de sistemas florestais. Os índices de diversidade variam ao longo dos meses e ao longo dos estratos do sistema solo-serapilheira revelando papel sazonal e espacial na diversidade da macrofauna. A diversidade e composição da macrofauna com seus papéis funcionais indicam um solo saudável.

AGRADECIMENTOS: Ao Gerson Rodrigues, por permitir a execução deste estudo em sua propriedade. Ao MEC através do Programa PROEXT, pelo apoio financeiro.

\section{REFERÊNCIAS}

ANDERSON, J. M.; INGRAM, J. S. I.. Tropical soil biology and fertility: a handbook of methods. 2 ed. Wallingford: $C A B$ International, 1993.

ANDERSON, J. M.. Why should we care about soil fauna? Pesquisa Agropecuária Brasileira, Brasília, v.44, n.8, p.835842, 2009. DOI: http://dx.doi.org/10.1590/S0100204X2009000800006

AQUINO, A. M.; SILVA, R. F.; MERCANTE, F. M.; CORREIA, M. E. F.; GUIMARÃES, M. F.; LAVELLE, P.. Invertebrate soil macrofauna under different ground cover plants in the notill system in the Cerrado. Europian Journal of Soil Biology, v.44, n.2, p.191-197, 2008. DOI:

https://doi.org/10.1016/i.ejsobi.2007.05.001

ARAUJO, V. F. P.; SILVA, M. P.; VASCONCELLOS, A.. Soilsampled termites in Two Contrasting Ecosystems within the Semiarid Domain in Northeastern Brazil: Abundance, Biomass, and Seasonal Influences. Sociobiology, Feira de Santana, v.62, n.1, p.70-75, 2015. DOI:

http://dx.doi.org/10.13102/sociobiology.v62i1.70-75
ASHFORD, O. S.; FOSTER, W.A .; TURNER, B. L.; SAYER, E. J.; SUTCLIFFE, L.; TANNER, E. V. J.. Litter manipulation and the soil arthropod community in a lowland tropical rainforest. Soil Biology \& Biochemistry, v.62, p.5-12, 2013. DOI: https://doi.org/10.1016/j.soilbio.2013.03.001

AYRES, M.; AYRES, J. M.; AYRES, D. L.; SANTOS, A. S.. Bioestat 5.0: Aplicações estatísticas nas áreas das ciências biomédicas. Belém: Mamirauá, 2007.

BARROS, E.; MATHIEU, J.; CORAL, S.; NASCIMENTO, A. L.; LAVELLE, P.. Comunidades da macrofauna do solo na Amazônia brasileira. In: MOREIRA, F. M. S.; SIQUEIRA, J. O.; BRUSSAARD, L.. Biodiversidade do solo em ecossistemas brasileiros. Lavras: UFLA, 2008. p.171-191.

EMBRAPA. Mapa de solos do Estado do Paraná. Rio de Janeiro: Embrapa Solos Floresta, 2007.

FEENER JR, D. H.; ORR, M. R.; WACKFORD, K. M.; LONGO, J. M.; BENSON, W. W.; GILBERT, L. E.. Geographic variation in resource dominance-discovery in brazilian ant communities. 
Ecology, Washington, v.89, n.7, p.1824-1836, 2008. DOI: https://doi.org/10.1890/07-0659.1

HUTCHESON, K.. A Test for Comparing Diversities based on the Shannon Formula. Journal of Theoretical Biology, v.29, n.1, p.151-154, 1970. DOI: https://doi.org/10.1016/00225193(70)90124-4

LAVELLE, P.; DECAËNS, T.; AUBERT, M.; BAROT, S.; BLOUIN, M.; BUREAU, F.; MARGERIE, P.; MORA, P.; ROSSI, J. P.. Soil invertebrates and ecosystem services. European Journal of Soil Biology, v.42, s.1, p.S3-S15, 2006. DOI: https://doi.org/10.1016/j.ejsobi.2006.10.002

LIMA, A. C. R.; BRUSSAARD, L.. Earthworms as soil quality indicators: local and scientific knowledge in rice management systems. Acta Zoológica Mexicana, Veracruz, v.26, n.2, p.109-116, 2010

LIMA, A. C. R.; RODRÍGUEZ, C.. Earthworm diversity from Rio Grande do Sul, Brazil, with a new native Criodrilid genus and species (Oligochaeta: Criodrilidae). Megadrilogica, v.11, n.2, p.9-18, 2007

LIMA, S. S.; AQUINO, A. M.; LEITE, L. F. C.; VELÁSQUEZ, E.; LAVELLE, P.. Relação entre macrofauna edáfica e atributos químicos do solo em diferentes agroecossistemas. Pesquisa Agropecuária Brasileira, Brasília, v.45, n.3, p.322-331, 2010. DOI: http://dx.doi.org/10.1590/S0100-204X2010000300013

MACHADO, E. L. M.; HIGASHIKAWA, E. M.; MACEDO, R. L. G.; VENTURIN, N.; NAVES, M. L.; GOMES, J. E.. Análise da diversidade entre sistemas agroflorestais em assentamentos rurais no sul da Bahia. Revista Científica Eletrônica de Engenharia Florestal, v.5, n.1, p.1-14, 2005.

MARQUES, D. M.; SILVA, A. B.; SILVA, L. M.; MOREIRA, E. A.; PINTO, G. S.. Macrofauna edáfica em diferentes coberturas vegetais. Bioscience Journal, Uberlândia, v.30, n.5, p.15881597, 2014

MAYER, P. M.; TUNNEL, S. J.; ENGLE, D. M.; JORGENSEN, E. E.; NUNN, P. V.. Invasive Grass Alters Litter Decomposition by Influencing Macrodetritivores. Ecosystems, v.8, n.2, p.200-209, 2005. DOI: https://doi.org/10.1007/s10021-004$\underline{0018-x}$

MELO, F. V.; BROWN, G. G.; CONSTANTINO, R.; LOUZADA, J. N. C.; LUIZÃO, F. J.; MORAIS, J. W.; ZANETTI, R.. A importância da meso e macrofauna do solo na fertilidade e como bioindicadores. Boletim Informativo da Sociedade Brasileira de Ciência do Solo, Viçosa, v.34, n.1, p.39-43, 2009.

MENEZES, C. E. G.; CORREIA, M. E. F.; PEREIRA, M. G.; BATISTA, I.; ROGRIGUES, K. M.; COUTO, W. H.; ANJOS, L. H. C.; OLIVEIRA, Í. P.. Macrofauna edáfica em estádios sucessionais de Floresta Estacional Semidecidual e pastagem mista em Pinheiral (RJ). Revista Brasileira de Ciências do
Solo, Viçosa, v.33, n.6, p.1647-1656, 2009. DOI:

http://dx.doi.org/10.1590/S0100-06832009000600013

MINEROPAR. Minerais Do Paraná. Atlas geomorfológico do Estado do Paraná. Escala Base: 1:250.000. Modelos Reduzidos: 1:500.000. Curitiba, 2006.

MOÇO, M. K. S.; GAMA-RODRIGUES, E. F.; GAMARODRIGUES, A. C.; CORREIA, M. E. F.. Caracterização da fauna edáfica em diferentes coberturas vegetais na região Norte Fluminense. Revista Brasileira de Ciência do Solo, Viçosa, v.29, n.4, p.555-564, 2005. DOI: http://dx.doi.org/10.1590/S0100-06832005000400008

OLIVEIRA-COSTA, J.; OLVEIRA, R. G.; BASTOS, C. S.. Diptera Calliphoridae de importância forense no município do Rio de Janeiro. Revista Eletrônica Novo Enfoque, v.16, n.16, p.41$52,2013$.

PINHEIRO, F. J.; MARTINS, C. M.; FIALHO, J. S.; CORREIA, M. E. F.; CASCON, P.. Caracterização da macrofauna edáfica na interface solo serapilheira em uma área de caatinga do nordeste brasileiro. Enciclopédia biosfera, Jandaia, v.10, n.19, p.2294-2307, 2014.

RUIZ, N.; LAVELLE, P.; JIMENEZ, J.. Soil Macrofauna Field Manual: Technical Level. Food and Agriculture Organisation of the United Nations, 2008.

SCHIEDECK, G.; SCHIAVON, G. A.; MAYER, F. A.; LIMA, A. C. R. Percepção de agricultores sobre o papel das minhocas nos agroecossistemas. Revista Brasileira de Agroecologia, Pelotas, v.4, n.2, p.856-859, 2009.

SILVA, J.; JUCKSCH, I.; FERES, C. I. M. A.; TAVARES, R. C. Fauna do solo em sistemas de manejo com café. Soil faunal in management systems with coffee. Journal of Biotechnology and Biodiversity, Gurupi, v.3, n.2, p.59-71, 2012.

SILVA, R. F.; AQUINO, A. M.; MERCANTE, F. M.; GUIMARÃES, $M$. F.. Macrofauna invertebrada do solo sob diferentes sistemas de produção em Latossolo da Região do Cerrado. Pesquisa Agropecuária Brasileira, Brasília, v.41, n.4, p.697704, 2006. DOI: http://dx.doi.org/10.1590/S0100204X2006000400022

SOUZA, A. K. P.; HERNÁNDEZ, M. I. M.; MARTINS, C. F. Riqueza, abundância e diversidade de Euglossina (Hymenoptera, Apidae) em três áreas da Reserva Biológica Guaribas, Paraíba, Brasil. Revista Brasileira de Zoologia, Curitiba, v.22, n.2, p.320-325, 2005. DOI: http://dx.doi.org/10.1590/S0101-81752005000200004

TEIXEIRA, C. C. L.; HOFFMANN, M.; SILVA-FILHO, G.. Comunidade de Coleoptera de solo em remanescente de Mata Atlântica no estado do Rio de Janeiro. Biota Neotropica, Campinas, v.9, n.4, p.91-95, 2009.

A CBPC - Companhia Brasileira de Produção Científica (CNPJ: 11.221.422/0001-03) detém os direitos materiais desta publicação. Os direitos referem-se à publicação do trabalho em qualquer parte do mundo, incluindo os direitos às renovações, expansões e disseminações da contribuição, bem como outros direitos subsidiários. Todos os trabalhos publicados eletronicamente poderão posteriormente ser publicados em coletâneas impressas sob coordenação da Sustenere Publishing, da Companhia Brasileira de Produção Científica e seus parceiros autorizados. Os (as) autores (as) preservam os direitos autorais, mas não têm permissão para a publicação da contribuição em outro meio, impresso ou digital, em português ou em tradução. 